
surgery

\title{
Phase II study of chemoselection with docetaxel plus cisplatin and 5-fluorouracil induction chemotherapy and subsequent conversion surgery for locally advanced unresectable oesophageal cancer
}

Tomoya Yokota*, ${ }^{*}$, Ken Kato ${ }^{2}$, Yasuo Hamamoto ${ }^{3}$, Yasuhiro Tsubosa ${ }^{4}$, Hirofumi Ogawa ${ }^{5}$, Yoshinori Ito ${ }^{6}$, Hiroki Hara ${ }^{7}$, Takashi Ura ${ }^{8}$, Takashi Kojima ${ }^{9}$, Keisho Chin $^{10}$, Shuichi Hironaka ${ }^{11}$, Takayuki Kii ${ }^{12}$, Yasushi Kojima ${ }^{13}$, Yasunori Akutsu ${ }^{14}$, Hisayuki Matsushita ${ }^{15}$, Kentaro Kawakami ${ }^{16}$, Keita Mori ${ }^{17}$, Yushi Nagai ${ }^{18}$, Chika Asami ${ }^{18}$ and Yuko Kitagawa ${ }^{19}$

Background: The standard treatment for locally advanced unresectable squamous cell carcinoma (SCC) of the oesophagus is chemoradiation with cisplatin and 5-fluorouracil (CF-RT). This multicentre phase II trial assessed the safety and efficacy of chemoselection with docetaxel plus cisplatin and 5-fluorouracil (DCF) induction chemotherapy (ICT) and subsequent conversion surgery (CS) for initially unresectable locally advanced SCC of the oesophagus.

Methods: Patients with clinical T4 and/or unresectable supraclavicular lymph node metastasis were eligible. Treatment started with three cycles of DCF-ICT, followed by CS if resectable, or by CF-RT if unresectable. The resectability was re-evaluated at 30-40 Gy of CF-RT, followed by CS if resectable, or by completion of 60 Gy of CF-RT. If resectable after CF-RT, CS was performed. The primary end point was 1-year overall survival (OS).

Results: From April 2013 to July 2014, 48 patients were enrolled. CS was performed in 41.7\% ( $n=20)$, including DCF-CS $(n=18)$, DCF-CF-RT40Gy-CS $(n=1)$, and DCF-CF-RT60Gy-CS $(n=1)$. R0 resection was confirmed in 19 patients (39.6\%). Grade $\geqslant 3$ postoperative complications included one event each of recurrent laryngeal nerve palsy, lung infection, wound infection, pulmonary fistula, and dysphagia; but no serious postoperative complications were observed in patients undergoing CS. Clinical complete response after CF-RT was confirmed in 4 patients (8.3\%). The estimated 1-year OS was 67.9\% and lower limit of $80 \%$ confidence interval was $59.7 \%$. There was one treatment-related death in patient receiving DCF-CF-RT60Gy.

Conclusions: Chemoselection with DCF-ICT followed by CS as a multidisciplinary treatment strategy showed promising signs of tolerability and efficacy in patients with locally advanced unresectable SCC of the oesophagus.

Standard therapy for locally advanced but resectable squamous cell carcinoma (SCC) of the oesophagus is neoadjuvant chemotherapy or chemoradiotherapy (CRT) followed by surgery (van Hagen et al, 2012) or definitive CRT. However, oesophageal cancers have a high tendency to invade adjacent organs, such as the trachea, lungs, heart, and aorta, owing to the lack of serosa in the oesophagus (Rustgi and El-Serag, 2014). Clinical T4 oesophageal cancer is defined by tumours' invasion of adjacent organs. The patients with clinical T4 disease or M1 lymph node (M1Lym) metastasis have not been considered for surgery, because curative resection is thought to be unfeasible. Furthermore,

*Correspondence: Dr T Yokota; E-mail: t.yokota@scchr.jp

Received 14 September 2016; accepted 3 October 2016; published online 3 November 2016

(c) 2016 Cancer Research UK. All rights reserved 0007-0920/16 
there is concern regarding increase in major perioperative complications if oesophagectomy is performed after CRT (Stahl et al, 2005; Bedenne et al, 2007). Therefore, definitive CRT without planned oesophagectomy is currently considered standard treatment for locally advanced unresectable oesophageal cancer (Ohtsu et al, 1999; Stahl et al, 2013; Shinoda et al, 2015; NCCN Clinical Practice Guidelines in Oncology, 2016). However, clinical T4 oesophageal cancer has still an unfavourable prognosis even after CRT (Chak et al, 1995).

Docetaxel plus cisplatin and 5-fluorouracil (DCF) induction chemotherapy (ICT) has been shown to elicit a good response and improve outcomes in locally advanced oesophageal cancer. A phase 2 study suggested that preoperative DCF was well tolerated and had highly promising antitumour activity as intensive neoadjuvant chemotherapy in patients with locally advanced resectable oesophageal cancer (Hara et al, 2013). Furthermore, a retrospective study using cohorts with locally advanced oesophageal cancer with suspected invasion to adjacent organs demonstrated that DCF regimen was superior to CF regimen with regard to overall response, $\mathrm{R} 0$ resection rate, and histological therapeutic effects (Yokota et al, 2011).

Therefore, our treatment strategy was to perform curative surgery in patients who responded to ICT and experienced subsequent downstaging, aiming at cure. Chemoselection was defined as an approach to select patients who were eligible for curative surgery by using the response to ICT. We designated such surgery as conversion surgery (CS). This trial evaluated whether chemoselection with DCF-ICT and subsequent CS is safe and has survival benefits for initially unresectable locally advanced oesophageal cancer.

\section{MATERIALS AND METHODS}

Patients. Patients with histologically proven squamous cell, adenosquamous, or basaloid carcinoma of the thoracic oesophagus were eligible if they had any of the following conditions: clinical $\mathrm{T} 4$ cancer, at least one unresectable metastatic regional lymph node due to invasion into an adjacent organ, or computed tomographic (CT) evidence of M1Lym, such as fixed supraclavicular nodes. Regional lymph nodes were defined on the basis of criteria specified by the seventh edition of the Union for International Cancer Control TNM staging system (Sobin and Wittekind, 2009). Other eligibility criteria were as follows: no prior chemotherapy and/or RT for oesophageal or any other carcinoma, age 20-75 years, an Eastern Cooperative Oncology Group (ECOG) performance status (PS) of $0-1$, and adequate organ functioning. Patients with an oesophageal perforation, oesophageal fistula, tumour bleeding, distant organ metastases, serious complications, severe infection, or mental disorder, were excluded from the study. Written informed consent was obtained from all patients prior to enrollment. The study was approved by the institutional review board at each site. This study was registered with the UMIN Clinic Trials Registry (http://www.umin.ac.jp/ctr/), identification number UMIN000011089.

Overview of protocol treatment. Protocol therapy started with three cycles of DCF-ICT. If CT scan after the first or second cycle of DCF detected progressive disease (PD), the subsequent cycle was not permitted and switched to CRT. If curative resection was considered possible on CT scan review after the third cycle of DCF, the patient was scheduled for CS. If not resectable, definitive CRT was administered. Tumour was re-evaluated at 30-40 Gy during CRT. If resectable, irradiation was administered up to $40 \mathrm{~Gy}$ and switched to CS. If still unresectable, additional CRT with a total irradiation dose of $60 \mathrm{~Gy}$ was performed. If the remaining tumour was resectable after administration of $60 \mathrm{~Gy}$ CRT, CS was performed. Although resectability was judged by CT review, the evaluation of primary lesion by oesophagoscopy was mandatory before CS at any step (Figure 1).
Assessment of tumour. Oesophagoscopy and CT of the neck, chest, and abdomen were carried out prior to the study. Involvement of adjacent organs was determined by CT. Tumours were considered to be clinical T4 if they extended into the lumen or caused a deformity of the airway or if they were attached to the aorta at a contact angle of $>90^{\circ}$ in over three slices (Picus et al, 1983). Consultation with an institutional radiation oncologist was mandatory before enrollment to confirm that definitive RT was possible according to the protocol.

Response to DCF-ICT was evaluated according to Response Evaluation Criteria in Solid Tumors 1.1. Primary tumour response was evaluated by the modified criteria of the Japanese Society for Esophageal Diseases (Japan Esophageal Society, 2009) and categorised as complete response (CR), good partial response (PR), stable disease, or PD. Endoscopic CR was defined as disappearance of primary tumours without the presence of ulceration or malignant cells in biopsy specimens. Endoscopic good PR was defined as obvious morphological change, such as reduction or flattening of tumour or elevated lesion around the ulcer, along with healing of the ulcer floor. Clinical CR after CRT was defined as endoscopic CR and the disappearance of all visible lymph node metastases on CT imaging. An evaluation of clinical CR had to be confirmed by reassessment on endoscopy and CT $\geqslant 4$ weeks later.

DCF-ICT. DCF-ICT regimen consisted of intravenous docetaxel $70 \mathrm{mg} \mathrm{m}^{-2}$ and cisplatin $70 \mathrm{mg} \mathrm{m}^{-2}$ on day 1 and a continuous infusion of 5 -fluorouracil $750 \mathrm{mg} \mathrm{m}^{-2}$ day $^{-1}$ for 5 days. This regimen was repeated every 3 weeks until unacceptable toxicity, patient refusal, or disease progression, up to a maximum of 3 cycles. Prophylactic use of granulocyte colony-stimulating factor was allowed, and prophylactic use of ciprofloxacin on days 5-15 was mandatory.

Surgical procedure and evaluation of histological therapeutic effects. CS was performed within 8 weeks from the last administration of DCF-ICT or CRT. All participating surgeons were allowed to use any operative approach and technique, with regard to thoracotomy or laparotomy, extent of lymphadenectomy, resection margin, reconstruction methods, and other synchronous surgery. To pursue surgical R0 resection, clinically positive lymph nodes diagnosed at baseline, including supraclavicular fossa nodes, were to be resected as much as possible. If tumour invade into adjacent organ, the simultaneous resection of the adjacent organ is optional according to the physicians' discretion. Evaluations of residual tumour (R), pathological curability, and histological therapeutic effects were classified according to the guidelines for clinical and pathological studies on carcinoma of the oesophagus (Japan Esophageal Society, 2009). Evaluations of R were classified as follows: R0: no residual tumour, R1: suspicious of residual tumour or microscopic residual tumour, and R2: macroscopic residual tumour. Evaluations of pathological curability were classified as follows: CurA: pStage 0-III with R0 and the nodal dissection area $(\mathrm{pD})$ larger than the extent of nodal metastasis $(\mathrm{pN})$, CurC: pathological residual tumour, and CurB: neither CurA nor CurC. Classification of the extent of lymph node dissection is based on nodes completely dissected. If node dissection is incomplete, the classification rank recorded is one grade lower. Histological therapeutic effects were classified as follows: grade 3: complete disappearance of viable cancer cells in the tumour bed (pathological CR); grade 2: disappearance of greater than twothirds of viable cancer cells; grade $1 \mathrm{~b}$ : disappearance of less than two-thirds but greater than one-thirds of viable cancer cells; and grade 1a: disappearance of less than one-thirds of viable cancer cells. The procedure after $\mathrm{R} 1$ or $\mathrm{R} 2$ resection was not provided by the protocol, and any of postoperative treatment was permitted according to the physicians' discretion. 


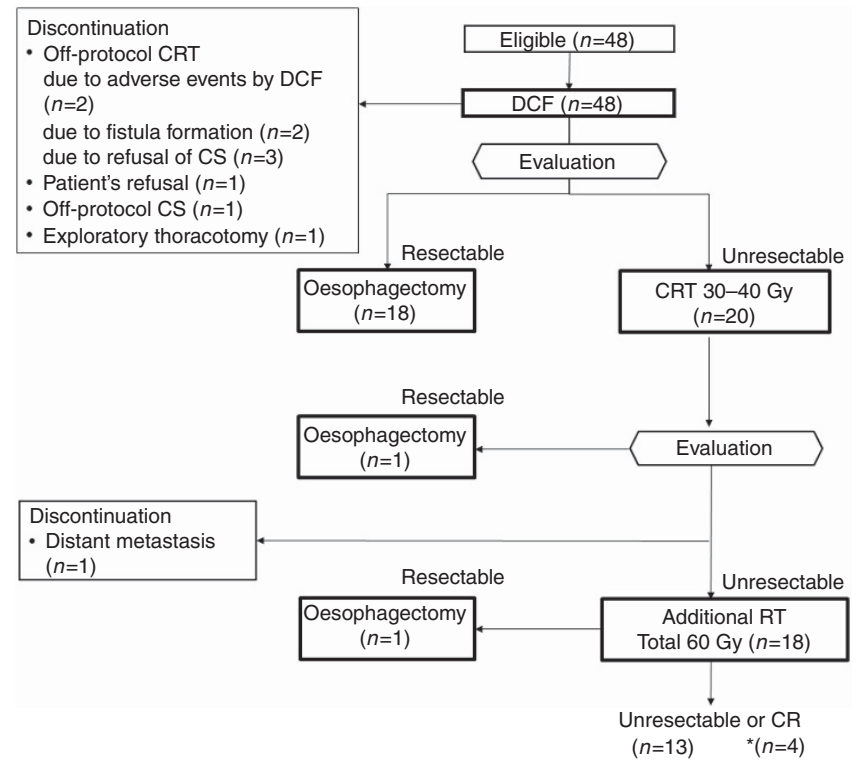

Figure 1. CONSORT flow diagram. *In all, $23.5 \%$ (4 out of 17) of patients who were ineligible for CS achieved CR after completion of $\mathrm{CRT}$. CR = complete response; $\mathrm{CRT}=$ chemoradiotherapy; $\mathrm{CS}=$ conversion surgery; DCF $=$ docetaxel plus cisplatin and 5-fluorouracil; RT = radiotherapy.

Chemoradiotherapy. Chemotherapy consisted of $70 \mathrm{mg} \mathrm{m}^{-2}$ cisplatin given on days 1 and 29 combined with a continuous infusion of $700 \mathrm{mg} \mathrm{m}^{-2}$ 5-FU given on days 1-4 and 29-32. Radiotherapy consisted of $60 \mathrm{~Gy}$ with a daily dose of $2 \mathrm{~Gy}$ and was delivered with 6-10 MV X-rays. Three-dimensional conformal radiation therapy with a CT stimulator was used. Clinically positive lymph nodes diagnosed at baseline, including supraclavicular fossa nodes, were to be irradiated, even though they drastically reduced in size by responding to DCF-ICT. Gross tumour volume (GTV) was determined by pretreatment CT and oesophagoscopy. Clinical target volume (CTV) included GTV with a craniocaudal margin of $2 \mathrm{~cm}$ at the primary site and with no margin in lymph node metastases. The planning target volume was defined by adding margins to the CTV at the discretion of the treating radiation oncologists (typically $0.5-1 \mathrm{~cm}$ for lateral margins and $1-2 \mathrm{~cm}$ for craniocaudal margins, depending on respiratory motion and patient immobilisation technique). Because the target volume was always large in very advanced oesophageal cancer, no prophylactic irradiation of lymph node area was performed.

Assessment of adverse events. Haematological and nonhaematological toxicity were assessed according to the Common Terminology Criteria of Adverse Events version 4.0, and the highest grade occurring at any time during ICT was reported. Perioperative complications were defined as those occurring from the time of surgery to first discharge from hospital. Late complications after surgery were defined as those occurring after the first discharge. Late complications after CRT were defined as those occurring $>91$ days after the CRT initiation.

Statistical analysis. Case reporting forms for each patient were collected from Clinical Data Management, Clinical Research Data Center, National Cancer Center Hospital, Tokyo, Japan.

The primary end point was 1-year survival rate, and the secondary end points were progression-free survival (PFS), overall response rate in DCF-ICT, R0 resection rate, CR rate in CRT, adverse events associated with DCF-ICT, surgery-related complications, treatment-related death, and late complications after CRT.

In JCOG 0303 trial, 1-year survival rate in the arm of standard dose CF-RT was 55.9\% (Shinoda et al, 2015). Therefore, we assumed a null hypothesis with a 50\% 1-year survival rate for our treatment strategy and expected a 1-year survival rate of $65 \%$. With $80 \%$ power and a one-sided type 1 error of $5 \%$, the minimum number of patients required to evaluate the primary end point was 47. Assuming a drop-out rate, we calculated the required total sample size as 48 patients. PFS was calculated from the date of registration to disease relapse or censored at last confirmation of survival. Overall survival (OS) was determined from the date of registration to the date of death from any causes or the last confirmation of survival. OS and PFS were estimated by using the Kaplan-Meier method, and curves were compared using a log-rank test. We carried out all the analyses using $\mathrm{R}$ version 3.1.3 (The R Foundation for Statistical Computing, Vienna, Austria). Two-sided $P$-values $<0.05$ were considered statistically significant.

\section{RESULTS}

Patient characteristics. This trial was started in April 2013 and closed in July 2014. A total of 48 patients were enrolled from 12 institutions in Japan. Baseline characteristics of all 48 patients are listed in Table 1 . Most patients were men (85\%), and the median age was 66 years (range, 47-74 years). All treated patients had an ECOG PS of 0 or 1. Histopathological findings from biopsy specimens of 47 patients (98\%) indicated SCC. Forty-two of 48 patients $(88 \%)$ were of $\mathrm{T} 4 \mathrm{~b}$ status.

Treatment profile. An accrual and treatment profile is shown in Figure 1. After the first or second cycle of DCF, the subsequent cycle of DCF was switched to CRT in four patients owing to PD. Three patients did not receive the second cycle of DCF. Of these, two patients received subsequent off-protocol CRT owing to adverse events. One patient refused to continue subsequent treatment, which was not related to adverse events. Two patients did not receive the third cycle of DCF because of oesophageal fistula formation.

After the third cycle of DCF, CS was performed in 18 patients. Five patients discontinued protocol treatment after the third cycle of DCF, even though curative resection was considered possible in all these patients. Of these, four patients refused CS, including three patients who received subsequent off-protocol CRT and one patient who underwent off-protocol CS after changing his mind. CS was not performed owing to unresectable findings on exploratory thoracotomy in one patient. The remaining 16 patients received subsequent CRT because curative resection was considered impossible.

One patient underwent CS after $40 \mathrm{~Gy}$ of CRT. One patient terminated CRT because of distant metastasis detected at reevaluation at $30-40 \mathrm{~Gy}$ during CRT. Thus the remaining 18 patients completed $60 \mathrm{~Gy}$ of CRT. One patient underwent CS for residual disease after $60 \mathrm{~Gy}$ of CRT. Overall, curative resection was considered possible in 25 patients $(52.1 \%)$, including 23 patients after DCF, 1 patient after 40 Gy of CRT, and 1 patient after 60 Gy of CRT. Twenty patients underwent CS as protocol treatment.

Efficacy outcomes. PR was observed in 15 patients treated with DCF and no CR was observed, giving an overall response rate of $31.3 \%$. By the subsequent treatment with CS, R0 resection was achieved in 19 patients (Table 2). This suggests that an overall R0 resection rate is $39.6 \%$ (19 out of 48 ) and that $\mathrm{R} 0$ resection was achieved in $95.0 \%$ of 20 patients who received on-protocol CS. However, one patient who underwent CS after 60 Gy of CRT was pathologically proven to be R1 resection after surgery. Histopathological CR (grade 3) was achieved in 4 out of 20 (20\%) of patients. Grade 2, 1b, and 1a responses were seen in 8 out of 20 (40\%), 6 out of $20(30 \%)$, and 2 out of $20(10 \%)$ of patients, respectively. Four of the 17 patients who completed 60 Gy of CRT without receiving CS (Figure 1) achieved clinical CR. Taken 
together, 23 patients (47.9\%) were successfully cured either by $\mathrm{R} 0$ resection or CRT.

Survival. The median follow-up period in patients surviving without death was 22.6 months (range, 1.1-33.5 months). It was impossible to thoroughly follow-up one patient whose follow-up period was 33 days because of refusal of subsequent treatment and hospital transfer. The median OS for all patients was not reached. A point estimate of 1-year survival rate was $67.9 \%$ and the lower limit of $80 \%$ confidence interval was $59.7 \%$ (Figure $2 \mathrm{~A}$ ). The OS for patients who underwent R0 resection was significantly longer than those who did not undergo R0 resection (median survival time: not reached $v s 8.0$ months, $P=0.003$ ) (Supplementary Data $S 1$ ). Of the 29 patients who did not undergo R0 resection, 16 patients died. The cause of their death was all progression of cancer. Of these, 4 patients died of bleeding from a primary lesion within 11 months after initiation of treatment. The median PFS for all patients was 17.6 months (Figure 2B).

Adverse events associated with DCF-ICT. Overall toxicities during DCF-ICT are listed in Table 3. The major toxicities were leukopenia and neutropenia. Despite antibiotic prophylaxis, febrile neutropenia occurred in $11(22.9 \%)$ patients. Common nonhaematological adverse events above grade 3 were anorexia $(25.0 \%)$, diarrhoea $(10.4 \%)$, and nausea $(4.2 \%)$. There were no non-haematological adverse events of grade 4. Two patients developed treatment-related oesophageal fistula. No treatmentrelated deaths were observed during DCF-ICT.

Surgery-related complications. No intraoperative complications, such as thrombus, adjacent organ injury, myocardial infarction,

\begin{tabular}{|c|c|c|}
\hline Characteristics & $n$ & $\%$ \\
\hline Age (years) (median (range)) & \multicolumn{2}{|c|}{$66(47-74)$} \\
\hline \multicolumn{3}{|l|}{ Sex } \\
\hline Male & 41 & 85 \\
\hline Female & 7 & 15 \\
\hline \multicolumn{3}{|l|}{ Performance status (ECOG) } \\
\hline 0 & 28 & 58 \\
\hline 1 & 20 & 42 \\
\hline \multicolumn{3}{|l|}{ Histology } \\
\hline SCC & 47 & 98 \\
\hline Basaloid carcinoma & 1 & 2 \\
\hline \multicolumn{3}{|l|}{ Location of primary tumour in oesophagus } \\
\hline Upper & 13 & 27 \\
\hline Middle & 33 & 69 \\
\hline Lower & 2 & 4 \\
\hline \multicolumn{3}{|l|}{ Macroscopic classification of primary lesion } \\
\hline 1 & 5 & 10 \\
\hline 2 & 29 & 60 \\
\hline 3 & 12 & 25 \\
\hline $0-11 \mathrm{~b}$ & 1 & 2 \\
\hline $0-\|l a+0-\| l c$ & 1 & 2 \\
\hline \multicolumn{3}{|l|}{ Clinical TNM ${ }^{\mathrm{a}}$} \\
\hline T3 & 5 & 10 \\
\hline T4a & 1 & 2 \\
\hline $\mathrm{T} 4 \mathrm{~b}$ & 42 & 88 \\
\hline No & 4 & 8 \\
\hline N1 & 12 & 25 \\
\hline N2 & 22 & 46 \\
\hline N3 & 10 & 21 \\
\hline MO & 39 & 81 \\
\hline M1 & 9 & 19 \\
\hline Stage $3 c$ & 39 & 81 \\
\hline Stage 4 & 9 & 19 \\
\hline
\end{tabular}

and arrhythmia, were observed. Perioperative complications included recurrent laryngeal nerve palsy $(n=8)$, pleural effusion $(n=5)$, and lung infection $(n=3)$ (Table 4$)$. Grade 3 was noted for recurrent laryngeal nerve palsy, lung infection, wound infection, pulmonary fistula, and dysphagia, all of which were manageable. No grade 4 complications were observed. Late complications after surgery included dysphagia (grade $1, n=1$; grade $2, n=1$ ), grade 2 pneumonitis $(n=1)$, and grade 3 anastomotic stricture $(n=1)$. Overall, there was no mortality and no serious complications related to surgery.

Adverse events associated with CRT. Toxicities that occurred during CRT among the 18 treated patients are listed in Supplementary Data S2. Grade 3 haematological toxicities included leukopenia $(n=5,27.8 \%)$, neutropenia $(n=1,5.6 \%)$, and anaemia $(n=2,11.1 \%)$. Grade 3 non-haematological toxicities included oesophagitis, dysphasia, anorexia, and nausea ( $n=1$ each). A thromboembolic event occurred in 1 patient, which resolved with conservative management. No oesophageal fistula occurred. Late complications after CRT included pneumonitis (grade 1, $n=4$; grade $2, n=1)$, grade 1 lung abscess $(n=1)$, grade 3 oesophagitis $(n=1)$, and grade 3 anorexia $(n=1)$. There was one treatment-related death in a patient who received DCF-CFRT60Gy. The cause of death was respiratory bleeding 7 days after the termination of CF-RT.

\section{DISCUSSION}

This study is the first prospective trial investigating the efficacy of DCF-ICT aiming at down-staging and subsequent CS for initially unresectable locally advanced oesophageal cancer. The results revealed that an overall $\mathrm{R} 0$ resection was achieved in 39.6\%, and a point estimate of 1 -year survival rate was $67.7 \%$ with the lower limit of $80 \%$ confidence interval as $59.5 \%$, which was higher than the threshold of $50 \%$, suggesting that this trial is statistically positive. Furthermore, 1-year survival rate in this study was higher than that in the arm of standard dose CF-RT in JCOG 0303 trial. These results suggest that DCF-ICT is sufficiently powerful preoperative treatment to result in high rate of curative resection and 1-year survival even in patients with initially unresectable clinical T4 oesophageal cancer.

In our protocol, CS was also permitted during or after completion of definitive CRT, even if the tumour was not converted to be curative after DCF-ICT. However, interestingly, of the 20 patients who underwent oesophagectomy, 18 patients (90\%) underwent CS after completion of DCF-ICT, and the other 2 patients did so during and after CRT. This suggests that patients who were able to undergo CS were mostly 'chemo-selected'. On the other hand, only $23.5 \%$ ( 4 out of 17 ) of patients who were ineligible

Table 2. Residual tumour and pathological curability $\left(n=20^{\mathrm{a}}\right)$

\begin{tabular}{|l|c|c|c|c|c|c|}
\hline \multicolumn{2}{|c|}{} & \multicolumn{3}{c|}{ Curability } \\
\hline $\begin{array}{l}\text { Residual } \\
\text { tumour }\end{array}$ & $\begin{array}{c}\text { Extent of } \\
\text { lymph node } \\
\text { dissection }\end{array}$ & $\begin{array}{c}\text { Resection } \\
\text { margin }\end{array}$ & CurA & CurB & CurC & All \\
\hline \multirow{3}{*}{ R0 } & D1 & PMODM0 & 1 & 0 & 0 & 1 \\
& D2 & PMODM0 & 2 & 1 & 0 & 3 \\
& D3 & PMODM0 & 12 & 2 & 0 & 14 \\
& PMXDM0 & 1 & 0 & 0 & 1 \\
\hline R1 & D2 & PM1DM0 & 0 & 0 & 1 & 1 \\
\hline Abbreviations: DM = distal margin; PM= proximal margin. Residual tumour was recorded in \\
accordance with the tenth edition of the Japanese Classification of Oesophageal Cancer. \\
a Number of patients who received on-protocol conversion oesophagectomy.
\end{tabular}


for CS achieved CR after completion of CRT. This may be because chemo- or CRT-resistant population selected by DCF-ICT was subsequently treated by CRT. However, it is noteworthy that $47.9 \%$ (19 patients with R0 resection and 4 patients with clinical CR in all 23 out of the 48) of patients with unresectable oesophageal cancer became free of disease using a tri-modality strategy with chemotherapy, radiotherapy, and surgery.

A

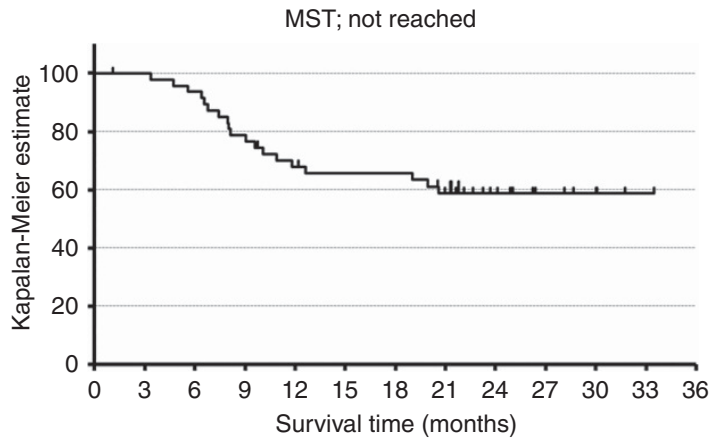

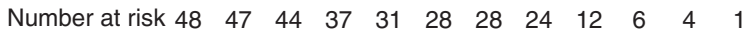

B

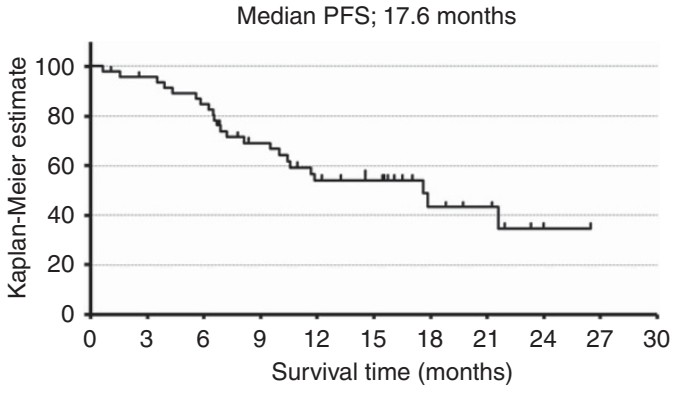

$\begin{array}{lllllllllll}\text { Number at risk } 48 & 44 & 39 & 29 & 23 & 23 & 21 & 16 & 10 & 6 & 4\end{array}$

Figure 2. Kaplan-Meier plot. Kaplan-Meier plot showing (A) overall survival and (B) progression-free survival of all patients $(n=48)$. $\mathrm{MST}=$ median survival time; PFS $=$ progression-free survival.
Furthermore, OS for patients who underwent R0 resection was significantly longer than those who did not undergo R0 resection, and all patients were alive for $>1$ year after the surgery (Supplementary Data S1). This suggests that DCF chemoselection may identify long-term survivors and contribute to overall improvement of survival outcomes in locally advanced oesophageal SCC. Several reports suggest that the addition of ICT to locoregional treatment significantly reduces the risk of distant metastases compared with locoregional treatment alone in patients with SCC of head and neck (Pignon et al, 2009). Therefore, we also expect that DCF-ICT could reduce the risk of distant metastases. Further analyses of disease recurrence or distant metastasis patterns are required by longer follow-up of survival.

It has been controversial whether curative surgery has a role as a treatment modality performed after down-staging by CRT. Two randomised trials comparing preoperative CRT followed by surgery versus CRT alone have investigated the role of surgery in T3 and/or T4 diseases (Stahl et al, 2005; Bedenne et al, 2007). However, no OS benefit of adding surgery to CRT has been demonstrated. Furthermore, a significantly higher operative mortality rate was reported in both trials. For instance, CRT followed by surgery significantly increased treatment-related mortality compared with CRT alone ( $12.8 \%$ vs $3.5 \%$, respectively; $P=0.03)$ in the study by Stahl et al (2005). FFCD 9102 also reported that the 3-month mortality rate was $9.3 \%$ in surgery group compared with $0.8 \%$ in CRT alone group $(P=0.02)$. CRT adversely results in radiation-induced fibrosis on thoracic tissue together with the effects on patient performance status. These factors may be associated with increases in major perioperative complications, such as anastomotic leak and pulmonary events, leading to mortality after oesophagectomy (Markar et al, 2014). Thus there remain concerns regarding the potential risks of oesophagectomy after CRT.

However, dysphagia is the most common symptom among patients with locally advanced oesophageal cancer undergoing CRT alone. CRT-related mucosal injury can cause severe dysphagia (Coia et al, 1995), which worsens quality of life despite the achievement of a primary cure under organ preservation. To relieve progressive oesophageal stricture, a large number of patients undergoing CRT needs palliative procedures against dysphagia, such as endoscopic dilatation or stent placement. Indeed, there was

Table 3. Adverse events associated with DCF-ICT (CTCAE ver 4.0)

\begin{tabular}{|c|c|c|c|c|c|}
\hline & Grade 1, n (\%) & Grade 2, n (\%) & Grade 3, n (\%) & Grade 4, n (\%) & All, $n(\%)$ \\
\hline Anorexia & $10(20.8)$ & $13(27.1)$ & $12(25.0)$ & 0 & $35(72.9)$ \\
\hline Fatigue & $22(45.8)$ & $9(18.8)$ & $1(2.1)$ & 0 & $32(66.7)$ \\
\hline Diarrhoea & $5(10.4)$ & $9(18.8)$ & $5(10.4)$ & 0 & $19(39.6)$ \\
\hline Mucositis oral & $12(25.0)$ & $6(12.5)$ & 0 & 0 & $18(37.5)$ \\
\hline Dysphagia & $7(14.6)$ & $4(8.3)$ & $2(4.2)$ & 0 & $13(27.1)$ \\
\hline Vomiting & $8(16.7)$ & $1(2.1)$ & 0 & 0 & $9(18.8)$ \\
\hline Oesophageal fistula & 0 & 0 & $2(4.2)$ & 0 & $2(4.2)$ \\
\hline White blood cell decreased & $4(8.3)$ & 14 (29.2) & 15 (31.3) & $5(10.4)$ & $38(79.2)$ \\
\hline Neutrophil count decreased & $1(2.1)$ & $7(14.6)$ & $10(20.8)$ & $22(45.8)$ & $40(83.3)$ \\
\hline Anaemia & $29(60.4)$ & $14(29.2)$ & 0 & 0 & $43(89.6)$ \\
\hline Creatinine increased & $11(22.9)$ & $3(6.3)$ & 0 & 0 & $14(29.2)$ \\
\hline Hyponatremia & $22(45.8)$ & 0 & $6(12.5)$ & $1(2.1)$ & $29(60.4)$ \\
\hline
\end{tabular}


Table 4. Perioperative complications $\left(n=21^{a}\right)$ CTCAE ver 4.0

\begin{tabular}{|c|c|c|c|c|}
\hline & Grade 1, n (\%) & Grade 2, n (\%) & Grade 3, n (\%) & All, $n(\%)$ \\
\hline Recurrent laryngeal nerve palsy & $5(23.8)$ & $2(9.5)$ & $1(4.8)$ & $8(38.1)$ \\
\hline Pleural effusion & $4(19.0)$ & $1(4.8)$ & 0 & $5(23.8)$ \\
\hline Postoperative haemorrhage & 0 & $2(9.5)$ & 0 & $2(9.5)$ \\
\hline Wound infection & 0 & $1(4.8)$ & $1(4.8)$ & $2(9.5)$ \\
\hline Pulmonary fistula & 0 & $1(4.8)$ & $1(4.8)$ & $2(9.5)$ \\
\hline Dyspnoea & 0 & $1(4.8)$ & 0 & $1(4.8)$ \\
\hline
\end{tabular}

more frequent palliative procedures in patients treated by CRT alone than in those treated by CRT followed by surgery in FFCD 9102 (Bedenne et al, 2007). This result suggests that dysphagia and the ability for oral intake showed better improvement after oesophagectomy (Bonnetain et al, 2006). Furthermore, fistula formation and penetration into adjacent organs are caused by CRT during or after the treatment and can be the cause of treatmentrelated death. In JCOG 0303, oesophageal fistula associated with CRT developed in 22\% of the patients (Shinoda et al, 2015). Therefore, our treatment protocol positioned DCF chemotherapy as an upfront intensive modality without radiation for downstaging, considering the safety of subsequent CS. In this study, oesophageal fistula was observed in only two patients after completion of DCF-ICT. Furthermore, because the DCF regimen frequently induced neutropenia, good care should be taken about myelotoxicity. However, the frequency of myelotoxicity and febrile neutropenia were consistent with that in other studies (Posner et al, 2007; Vermorken et al, 2007), and these were manageable with prophylactic antibiotics. Importantly, no serious perioperative and late complications were observed in patients who underwent CS after DCF, suggesting that our treatment strategy is safe and manageable.

The first limitation of this study is that short-term survival rate was used as a primary end point. We expected that CS has a role in raising the tail of the Kaplan-Meier survival curve by increasing the number of long-term survivors. On the other hand, patients who failed to achieve clinical CR have a trend towards early death, owing to treatment-related death and PD. Therefore, we hypothesised that 1-year survival may determine subsequent long-term survival and be acceptable as a surrogacy for long survival in this phase II trial. Furthermore, the recent cancer treatment has been developed so rapidly that it is difficult to spend much time and infrastructure on the phase II trials. The third reason is that the primary end point used in JCOG 0303 trial, on which this study is based, was 1-year survival rate. However, we are currently following up the longer survival in all patients and planning to analyse 3-year survival rate in the future.

The second limitation is the reliability of pretreatment clinical diagnosis of $\mathrm{T}$ factor. Endoscopic ultrasonography (EUS) was not routinely performed to determine the depth of the primary tumour in the diagnosis of T4 disease. However, to validate the accuracy of clinical $\mathrm{T}$ diagnosis, a retrospective central review of imaging in all patients enrolled is currently being performed by physicians blinded to clinical data. Another criticism may be lack of the QOL assessment. In future clinical trials, appropriate combined use of CT and EUS and the QOL assessment should be outlined in the protocol.

In conclusion, chemoselection with DCF-ICT followed by CS showed promising signs of tolerability and efficacy in patients with locally advanced unresectable SCC of the thoracic oesophagus. Based on the results of our phase II trial, JCOG are planning a prospective randomised controlled trial to compare chemoselection with DCF-ICT followed by CS versus CF-RT as a standard treatment for locally advanced oesophageal cancers.

\section{ACKNOWLEDGEMENTS}

We thank Dr Takashi Ichimura, Dr Mariko Ogura, Dr Daisuke Takahari (Cancer Institute Hospital of Japanese Foundation for Cancer Research), Dr Fumihiko Kato, Dr Satoshi Matsuda (Keio University School of Medicine), Dr Natsuko Okita, Dr Satoru Iwasa, Dr Atsuo Takashima, Dr Yoshitaka Honma (National Cancer Center Hospital), Dr Takako Yoshii (Saitama Cancer Center), Dr Akiko Todaka, Dr Nozomu Machida, Dr Takahiro Tsushima (Shizuoka Cancer Center), Dr Keiko Minashi (Chiba Cancer Center), Dr Tetsushi Terasawa, Dr Hitoshi Nishitani (Osaka Medical College), and Dr Kazuhiko Yamada (National Center for Global Health and Medicine) for patient enrollment. We also thank Dr Hiroyasu Igaki for useful advice. This study was supported by a Grant-in-Aid for Cancer Research from the Ministry of Health, Labor and Welfare. This study was supported in part by the National Cancer Center Research and Development Fund (26-A-4).

\section{CONFLICT OF INTEREST}

The authors declare no conflict of interest.

\section{REFERENCES}

Bedenne L, Michel P, Bouché O, Milan C, Mariette C, Conroy T, Pezet D, Roullet B, Seitz JF, Herr JP, Paillot B, Arveux P, Bonnetain F, Binquet C (2007) Chemoradiation followed by surgery compared with chemoradiation alone in squamous cancer of the esophagus: FFCD 9102. J Clin Oncol 25: 1160-1168.

Bonnetain F, Bouché O, Michel P, Mariette C, Conroy T, Pezet D, Roullet B, Seitz JF, Paillot B, Arveux P, Milan C, Bedenne L (2006) A comparative longitudinal quality of life study using the Spitzer quality of life index in a randomized multicenter phase III trial (FFCD 9102): chemoradiation followed by surgery compared with chemoradiation alone in locally advanced squamous resectable thoracic esophageal cancer. Ann Oncol 17: 827-834.

Chak A, Canto M, Gerdes H, Lightdale CJ, Hawes RH, Wiersema MJ, Kallimanis G, Tio TL, Rice TW, Boyce Jr HW (1995) Prognosis of esophageal cancers preoperatively staged to be locally invasive (T4) by endoscopic ultrasound (EUS): a multicenter retrospective cohort study. Gastrointest Endosc 42: 501-506.

Coia LR, Myerson RJ, Tepper JE (1995) Late effects of radiation therapy on the gastrointestinal tract. Int J Radiat Oncol Biol Phys 31(5): 1213-1236.

Hara H, Tahara M, Daiko H, Kato K, Igaki H, Kadowaki S, Tanaka Y, Hamamoto Y, Matsushita H, Nagase M, Hosoya Y (2013) Phase II 
feasibility study of preoperative chemotherapy with docetaxel, cisplatin, and fluorouracil for esophageal squamous cell carcinoma. Cancer Sci 104: $1455-1460$.

Japan Esophageal Society (2009) Japanese Classification of Esophageal Cancer, 10th edition. Esophagus 6: 1-2571-94.

Markar SR, Karthikesalingam A, Penna M, Low DE (2014) Assessment of short-term clinical outcomes following salvage esophagectomy for the treatment of esophageal malignancy: systematic review and pooled analysis. Ann Surg Oncol 21: 922-931.

NCCN Clinical Practice Guidelines in Oncology (2016) Esophageal and Esophagogastric Junction Cancers, Version 2.2016. National Comprehensive Cancer Network: Fort Washington, PA, USA

Ohtsu A, Boku N, Muro K, Chin K, Muto M, Yoshida S, Satake M, Ishikura S, Ogino T, Miyata Y, Seki S, Kaneko K, Nakamura A (1999) Definitive chemoradiotherapy for T4 and/or M1 lymph node squamous cell carcinoma of the esophagus. J Clin Oncol 17: 2915-2921.

Picus D, Balfe DM, Koehler RE, Roper CL, Owen JW (1983) Computed tomography in the staging of esophageal carcinoma. Radiology 146: 433-438.

Pignon JP, le Maître A, Maillard E, Bourhis J. MACH-NC Collaborative Group (2009) Meta-analysis of chemotherapy in head and neck cancer (MACH-NC): an update on 93 randomised trials and 17346 patients. Radiother Oncol 92: 4-14.

Posner MR, Hershock DM, Blajman CR, Mickiewicz E, Winquist E, Gorbounova V, Tjulandin S, Shin DM, Cullen K, Ervin TJ, Murphy BA, Raez LE, Cohen RB, Spaulding M, Tishler RB, Roth B, Viroglio Rdel C, Venkatesan V, Romanov I, Agarwala S, Harter KW, Dugan M, Cmelak A, Markoe AM, Read PW, Steinbrenner L, Colevas AD, Norris Jr CM, Haddad RI. TAX 324 Study Group (2007) Cisplatin and fluorouracil alone or with docetaxel in head and neck cancer. N Engl J Med 357: 1705-1715.

Rustgi AK, El-Serag HB (2014) Esophageal carcinoma. N Engl J Med 371: 2499-2509.

Shinoda M, Ando N, Kato K, Ishikura S, Kato H, Tsubosa Y, Minashi K, Okabe H, Kimura Y, Kawano T, Kosugi S, Toh Y, Nakamura K, Fukuda H. Japan Clinical Oncology Group (2015) Randomized study of low-dose versus standard-dose chemoradiotherapy for unresectable esophageal squamous cell carcinoma (JCOG0303). Cancer Sci 106: 407-412.
Sobin LH, Wittekind C (2009) TNM Classification of Malignant Tumors (UICC). 7th edn. Wiley-Blackwell, UK. Available at: http://www.uicc.org/ resources/tnm.

Stahl M, Stuschke M, Lehmann N, Meyer HJ, Walz MK, Seeber S, Klump B, Budach W, Teichmann R, Schmitt M, Schmitt G, Franke C, Wilke H (2005) Chemoradiation with and without surgery in patients with locally advanced squamous cell carcinoma of the esophagus. J Clin Oncol 23: 2310-2317.

Stahl M, Mariette C, Haustermans K, Cervantes A, Arnold D. ESMO Guidelines Working Group (2013) Oesophageal cancer: ESMO Clinical Practice Guidelines for diagnosis, treatment and follow-up. Ann Oncol 24(Suppl 6): vi51-vi56.

van Hagen P, Hulshof MC, van Lanschot JJ, Steyerberg EW, van Berge Henegouwen MI, Wijnhoven BP, Richel DJ, Nieuwenhuijzen GA, Hospers GA, Bonenkamp JJ, Cuesta MA, Blaisse RJ, Busch OR, ten Kate FJ, Creemers GJ, Punt CJ, Plukker JT, Verheul HM, Spillenaar Bilgen EJ, van Dekken H, van der Sangen MJ, Rozema T, Biermann K, Beukema JC, Piet AH, van Rij CM, Reinders JG, Tilanus HW, van der Gaast A. CROSS Group (2012) Preoperative chemoradiotherapy for esophageal or junctional cancer. N Engl J Med 366: 2074-2084.

Vermorken JB, Remenar E, van Herpen C, Gorlia T, Mesia R, Degardin M, Stewart JS, Jelic S, Betka J, Preiss JH, van den Weyngaert D, Awada A, Cupissol D, Kienzer HR, Rey A, Desaunois I, Bernier J, Lefebvre JL. EORTC 24971/TAX 323 Study Group (2007) Cisplatin, fluorouracil, and docetaxel in unresectable head and neck cancer. N Engl J Med 357: 1695-1704.

Yokota T, Hatooka S, Ura T, Abe T, Takahari D, Shitara K, Nomura M, Kondo C, Mizota A, Yatabe Y, Shinoda M, Muro K (2011) Docetaxel plus 5-fluorouracil and cisplatin (DCF) induction chemotherapy for locally advanced borderline-resectable T4 esophageal cancer. Anticancer Res 31: 3535-3541.

This work is published under the standard license to publish agreement. After 12 months the work will become freely available and the license terms will switch to a Creative Commons AttributionNonCommercial-Share Alike 4.0 Unported License.

${ }^{1}$ Division of Gastrointestinal Oncology, Shizuoka Cancer Center, 1007 Shimonagakubo, Nagaizumi-cho, Sunto-gun, Shizuoka 4118777, Japan; ${ }^{2}$ Gastrointestinal Oncology Division, National Cancer Center Hospital, 5-1-1 Tsukiji, Chuo-ku, Tokyo 104-0045, Japan; ${ }^{3}$ Division of Gastroenterology \& Hepatology, Keio University School of Medicine, 35 Shinanomachi, Shinjuku-ku, Tokyo 160-8582, Japan; ${ }^{4}$ Division of Esophageal Surgery, Shizuoka Cancer Center, 1007 Shimonagakubo, Nagaizumi-cho, Sunto-gun, Shizuoka 411-8777, Japan; ${ }^{5}$ Division of Radiation Oncology, Shizuoka Cancer Center, 1007 Shimonagakubo, Nagaizumi-cho, Sunto-gun, Shizuoka 411-8777, Japan; ${ }^{6}$ Department of Radiation Oncology, National Cancer Center Hospital, 5-1-1 Tsukiji, Chuo-ku, Tokyo 104-0045, Japan; ' Department of Gastroenterology, Saitama Cancer Center, 818 Komuro, Inamachi, Kitaadachi-gun, Saitama 3620806, Japan; ${ }^{8}$ Department of Clinical Oncology, Aichi Cancer Center Hospital, 1-1 Kanokoden, Chikusa-ku, Nagoya 464-8681, Japan; ${ }^{9}$ Department of Gastroenterology, National Cancer Center Hospital East, 6-5-1, Kashiwanoha, Kashiwa, Chiba 277-8577, Japan; ${ }^{10}$ Department of Gastroenterology, Cancer Institute Hospital of Japanese Foundation for Cancer Research, 3-8-31 Ariake, Koto-ku, Tokyo 135-8550, Japan; ${ }^{11}$ Clinical Trial Promotion Department, Chiba Cancer Center, 666-2, Nitona-cho, Chuo-ku, Chiba 260-8717, Japan; ${ }^{12}$ Cancer Chemotherapy Center, Osaka Medical College Hospital, 2-7, Daigaku-cho, Takatsuki, Osaka 569-8686, Japan; ${ }^{13}$ Department of Gastroenterology, National Center for Global Health and Medicine, 1-21-1 Toyama, Shinjuku-ku, Tokyo 162-8640, Japan; ${ }^{14}$ Department of Frontier Surgery, Graduate School of Medicine, Chiba University, 1-8-1, Inohana, Chuo-ku, Chiba 260-8670, Japan; ${ }^{15}$ Department of Surgery, Tochigi Cancer Center, 4-9-13 Yonan, Utsunomiya, Tochigi 320-0834, Japan; ${ }^{16}$ Department of Medical Oncology, Tochigi Cancer Center, 4-9-13 Yonan, Utsunomiya, Tochigi 320-0834, Japan; ${ }^{17}$ Clinical Trial Coordination Office, Shizuoka Cancer Center, 1007 Shimonagakubo, Nagaizumi-cho, Sunto-gun, Shizuoka 411-8777, Japan; ${ }^{18}$ Clinical Data Management, Clinical Research Data Center, National Cancer Center Hospital, 5-1-1 Tsukiji, Chuo-ku, Tokyo 1040045, Japan and ${ }^{19}$ Department of Surgery, Keio University School of Medicine, 35 Shinanomachi, Shinjuku-ku, Tokyo 160-8582, Japan

Supplementary Information accompanies this paper on British Journal of Cancer website (http://www.nature.com/bjc) 